\title{
Surgical Outcome of Posterior Column Acetabular Fractures Treated By Modified Kocher Lagenbeck Approach
}

\author{
Dr. Sanjay A.K. ${ }^{1}$,Dr.U.Thyagarajan ${ }^{2}$,Dr.L.Senthil ${ }^{3}$ \\ Department of Orthopaedics/Sri Ramachandra Medical College And Hospital, Chennai, India
}

\begin{abstract}
In this study, post-operative functional outcome and post-operative complications in posterior column acetabular fractures treated by external rotators sparing kocher lagenbeck approach is studied. Patients and methods: This prospective and retrospective study includes 20 patients of posterior acetabular fractures with a mean age of 39.55 years (range 21-60), that had been treated by external rotator sparing Kocher lagenbeck approach from october 2010 to october 2013.

Results: Anatomic reduction and stable fixation of the fracture with less than $2 \mathrm{~mm}$ residual displacement was achieved in 18 of 20 hips. At the final follow up, the patients were evaluated clinically according to Merle d'Aubigne scoring system. The clinical results were excellent in 9, very good in 6, good in 3, fair in 1, and poor 1 hip.

Conclusion: The functional outcome was satisfactory in most of the cases and comparable with other larger series. Using quadratus sparing kocher lagenbeck approach resulted in good fracture reduction without approach related complications.
\end{abstract}

Keywords: Acetabular fracture; Modified posterior acetabular approach, Posterior column; modified; Short external rotator sparing.

\section{Introduction}

There is an increase in rate of acetabular fractures due to an increase in road traffic accidents. Fractures of the acetabulum occur primarily in young adults as a result of high-velocity accidents. Displacement of the fracture fragments leads to articular incongruity of the hip joint and results in an abnormal pressure distribution on the articular cartilage surface. This can lead to rapid breakdown of the cartilage surface, resulting in disabling the hip joint. Anatomic reduction and stable fixation of the fracture, such that the femoral head is concentrically reduced under an adequate portion of the weight-bearing dome of the acetabulum, is the treatment goal in these difficult fractures. With advances in imaging technologies, performing acetabular fracture surgery through smaller incisions is now possible. The Kocher-Langenbeck $(\mathrm{K}-\mathrm{L})$ approach is the most commonly used surgical exposure for the stabilization of acetabular fractures involving a displaced posterior component [1], [2] and [3]. With the $\mathrm{K}-\mathrm{L}$ approach access to transverse or posterior column fractures extending cranially to the angle of the greater sciatic notch can be difficult [4]. To address this shortcoming, both the osteotomy of the greater trochanter and $\mathrm{K}-\mathrm{L}$ modification in the proximal dissection have been described [4], [5] and [6]. The conventional $\mathrm{K}-\mathrm{L}$ approach involves splitting of the gluteus maximus and division of the short rotators of the hip [3]. To decrease approach related soft tissue trauma and lessen the incidence of heterotropic ossification, postoperative nerve injury, abductor weakness and joint stiffness; quadratus sparing $\mathrm{K}-\mathrm{L}$ approach have been described mostly in displaced, isolated posterior column fractures without marginal impaction [7] and [8]. We report our experience with short external rotators sparing modified posterior approach in both simple and complex acetabular fractures involving the posterior column or both to assess the functional outcome and incidence of complications in such patients.

\section{Materials And Methods}

From October 2013 toOctober 2016, 20 patients with displaced posterior column acetabular fractures were treated with open reduction and internal fixation using amodified K-L approach. The radiological and functional outcome was assessed. Merle D'Aubigne scoring system was used to evaluate the functional outcome.

\subsection{Surgical technique:}

The patients were placed in a lateral decubitus position on the radiolucent normal operating table under general/regionalanaesthesia. An incision beginning a handbreadth superior to posterior superior iliac spine on the iliac crest advancing laterally to the greater trochanter of femur and then curving posteriorly towards the gluteal fold was used. Gluteus maximus origin was not detached from the iliac crest; the plane between tensor fascia lata and gluteus maximus was used to reflect the gluteus maximus posteriorly. Distal part of the gluteus maximus insertion to femur was not divided. Then gluteus maximus was reflected posteriorly to provide 
exposure of the entire posterior pelvis and direct visualization of the sciatic nerve. Working in the superficial plane to external hip rotators, fracture site and when necessary the joint capsule were exposed between either gluteus medius and piriformis or piriformis and superior gemellus interval (superior portal). The cuff of short external rotators were left intact and plate bender was used for plate contouring. In most fractures gentle retraction of the gluteus medius to widen the superior portal is sufficient to reduce the displaced posterior fragment gently compressing it with a periosteal elevator. A curved $3.5 \mathrm{~mm}$ or $4.5 \mathrm{~mm}$ reconstruction plate was passed underneath the spared piriformis and short external rotators extending from lateral ischium to the inferior iliac wing compressing the fractured fragment [15]. After reduction of the fracture, fixation was achieved.

Passive range of motion exercises of the hip was applied to all patients just after the operation. Isotonic (hip flexor, abductor muscle groups) and isometric strengthening exercises (hip adductor and knee extensor muscle groups) were applied. Continuous passive motion (CPM) was applied to those having hip joint range of motion limitation. The patients were mobilised toe touch weight bearing with a walker or double crutches for 6-12 weeks.

The patients were evaluated clinically and radiographically at an immediate postoperative period of 2 days, 6 weeks, 3 months, 6 months, one year. Clinical grading was evaluated by Merle d'Aubigne scoring [10], [11] and [12]. The radiographic results were assessed according to the criteria described by Matta [11], [12] and [13]. A displacement of $1 \mathrm{~mm}$ or less was considered as anatomic, 2-3 mm as imperfect, and greater than $3 \mathrm{~mm}$ as poor [11], [12] and [13].

\section{Results}

In all cases, the modified K-L approach sparring the short external rotatorswas completely adequate to obtain fracture reduction and internal fixation. The average follow-up of all the patients was 8 months (range 3 to 9 months). 20 patients between the age group of 20 years till 60 years with an average age incidence of 39.55 years. We had 16 male patients and 4 female patients. In our series, right sided acetabular injuries were common (12 patients) compared to the left sided injuries (8 patients). In our study, we selected only posterior column acetabulam fractures. The average duration of surgery in patients operated after 2 weeks of injury was 235 mins , while in the patients operated earlier than 2 weeks was 186.4 mins. In 18 patients $(90 \%)$ congruent reduction was achieved intraoperatively. In 2 patientsincongruence persisted due to severe intra-articular communition. Few patients (30\%)required blood transfusion either during or after the surgery. The average number of transfused units of blood was 1.3. All the patients requiring column fixation were internally fixed with precontoured reconstruction plates.

The postoperative reduction was graded as excellent in 9 hips (45\%), very good in 6 (30\%), good in $3(15 \%)$, fair in $1(5 \%)$, and poor in 1 hip (5\%) according to the modified Merle d'Aubigne scoring. The radiographic results according to the criteria developed by Matta at the final follow-up were excellent in 12 hips $(60 \%)$, good in 6 hips (30\%), fair in 1 hip (5\%), and poor in one hip (5\%).

Functionally $75 \%$ fractures had a score labelled as excellent/very good.Avascular necrosis of the femoral head was not seen in any hips. In two of our cases, superficial local wound infection that was diagnosed in the early postoperative period was treated with antibiotics without debridement. There was no iatrogenic sciatic nerve palsy or heterotrophic ossification postoperatively. There were no cases of deep vein thrombosis or pulmonary embolism.

\section{Discussion}

The outcome of displaced acetabular fractures are influenced by many factors including the type of fracture, associated femoral head dislocation, timing of the surgery, the quality of reduction achieved during the surgery and the associated complication of the surgical approach used. A surgical approach which is less invasive, reduces the operative time, adequate working space for the fracture, minimizes the blood loss and will help in achieving optimal functional outcome. The aim of this study is to assess whether a modified less invasive K-L approach can help in management of most of the posterior wall/column fractures. The study also aimed at the radiological and functional outcome of patients operated with this approach.

In our series $90 \%$ patients had Excellent to good results, which is comparable to that reported in other studies. Patients between the age group of 20 years and 40 years had better outcome compared to patients above 50 years which was also reported by Libergall et al and Matta in their studies. This could be due to fact that complex fracture patterns were more common after 50 years of age and re-construction is difficult in osteoporotic and communited bone.

Management of displaced acetabular fractures requires adequate exposure and minimal morbidity of approach itself. An ideal approach would allow visualization of the column and joint surface with minimal complication. Extensile approach of hip had reported higher complication rate $(1,10)$. Alonso et al reported 53\% incidence of Heterotrophic ossification in triradiate approach and 86\% with extended ilio-femoral approach. Our approach to posterior column fractures which sparred the short external rotators dissection in K-L approach 
helps in reducing the excessive soft tissue dissection and the associated complications of deep infection, blood loss, heterotrophic ossification and gives good functional scores.
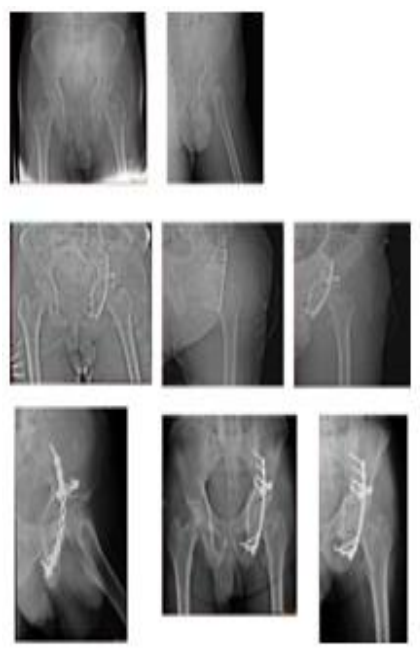

MM POST.OP

PRE-OP

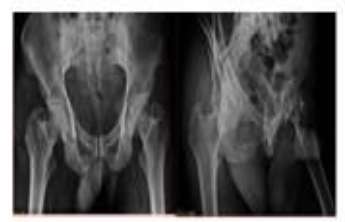

PRE.OP

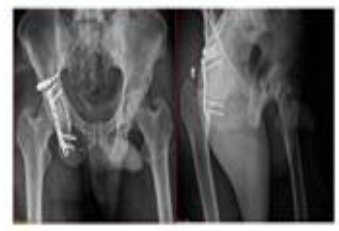

MM POST.OP

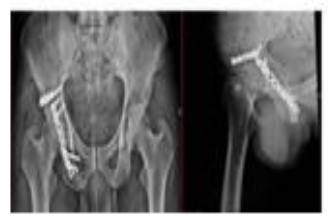

4 MONTHSFU

\section{Examples}

Name: Mr. SN Age: 45 yrsSex: male

Diagnosis: Bicolumnar fracture leftacetabulum with fracture left iliac wing.

Type C(AOClassification)

Results: Very good (Points 17)
Name:Mr. KNAge: 28 yrsSex : male

Diagnosis: fracture posterior column right acetabulum.

Type A (AO Classification)

Result: Good (Points 16)
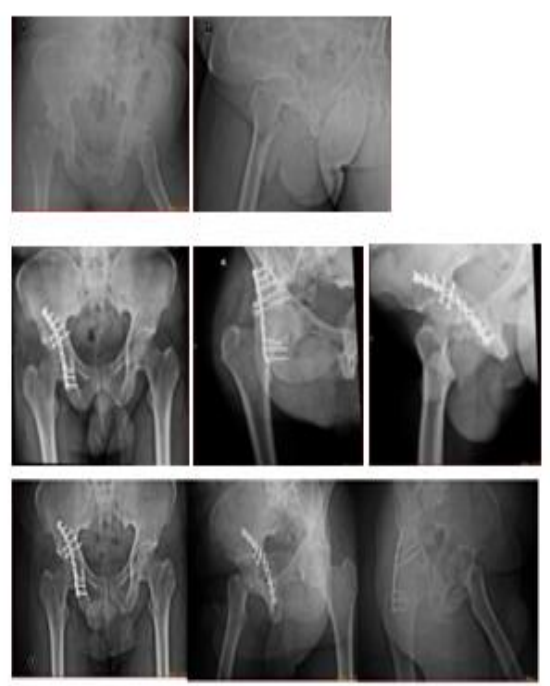

PRE-OP

IMM POST-OP

Name: Mrs. APAge : 34 yrsSex : male

Diagnosis : Fracture posterior column right acetabulum

Type A (AO Classification)

Results : Excellent(Point 18)

8 MONTHSFU 


\section{Conclusion}

The modified posterior approach decreases the operating time, results in good fracture reduction without approach related complications; thus seems to be a promising surgical procedure both in simple and selected complex posterior column acetabular fractures.

\section{References}

[1]. Judet R, Judet J, Letournel E. Fractures of the acetabulum: classification and surgical approaches for open reduction. J Bone Joint Surg Am 1964;46:1615- 75 .

[2]. Letournel E. Acetabulum fractures: classification and management. Clin Orthop Relat Res 1980;151:81-106.

[3]. Mehlman CT, Meiss L, DiPasquale TG. Hyphenated-history: the Kocher-Langenbeck surgical approach. J Orthop Trauma 2000;14:60-4.

[4]. Siebenrock KA, Gautier E, Ziran BH, Ganz R. Trochanteric flip osteotomy for cranial extension and muscle protection in acetabular fracture fixation using a Kocher-Langenbeck approach. J Orthop Trauma 2006;20(Suppl. 1):S52-6.

[5]. Moed BR. The modified Gibson posterior surgical approach to the acetabulum. J Orthop Trauma 2010;24:315-22.

[6]. Hadjicostas PT, Thielemann FW. The use of trochanteric slide osteotomy in the treatment of displaced acetabular fractures. Injury 2008;39:907-13.

[7]. Magu NK, Rohilla R, Arora S, More H, Modified. Kocher-Langenbeck approach for the stabilization of posterior wall fractures of the acetabulum. J Orthop Trauma 2011;25:243-9

[8]. Josten C, Trabold O. Modified "2-portal" Kocher-Langenbeck approach: a minimally-invasive procedure protecting the short external rotator muscles. J Orthop Trauma 2011;25:250-7.

[9]. Wey J, DiPasquale D, Levitt L, Quitkin H. Operative treatment of acetabular fractures through the extensile Henry approach. J Trauma 1999;46:255-60.

[10]. d'Aubigne RM, Postel M. Functional results of hip arthroplasty with acrylic prosthesis. J Bone Joint Surg Am 1954;36:451-75.

[11]. Matta JM, Mehne DK, Roffi R. Fractures of the acetabulum. Early results of a prospective study. Clin Orthop Relat Res 1986;205:241-50.

[12]. Matta JM. Operative treatment of acetabular fractures through the ilioinguinal approach: a 10-year perspective. Clin Orthop Relat Res 1994;305:10-9.

[13]. Matta JM. Fractures of the acetabulum: accuracy of reduction and clinical results in patients managed operatively within three weeks after the injury. J Bone Joint Surg Am 1996;78:1632-45.

[14]. Brooker AF, Bowerman JW, Robinson RA, Riley Jr LH. Ectopic ossification following total hip replacement: incidence and a method of classification. J Bone Joint Surg Am 1973;55:1629-32.

[15]. Ganz R, Gill TJ, Gautier E, Ganz K, Kru“ gel N, Berlemann U. Surgical dislocation of the adult hip a technique with full access to the femoral head and acetabulum without the risk of avascular necrosis. J Bone Joint Surg Br 2001;83:1119-24. 